\title{
PERFIL DOS ACIDENTES DE TRABALHO NA REGIÃO SUDOESTE DA BAHIA
}

\author{
Elizeu Cruz dos Santos*, Larissa Fernanda Pereira Gonçalves**, Camila Rego Amorim***, \\ Thalles da Costa Lobê Pereira ${ }^{* * *}$, Ana Cláudia Conceição da Silva ${ }^{* * * * *}$ \\ Autor correspondente: Ana Cláudia Conceição da Silva. - E-mail: anaclaudiacs@gmail.com \\ * Fisioterapeuta, Núcleo de Estudo e Pesquisa em Saúde do Trabalhador e Desigualdades em Saúde, Universidade Estadual \\ do Sudoeste da Bahia, Jequié, Bahia \\ ** Fisioterapeuta, Pesquisadora do Núcleo de Estudo e Pesquisa em Saúde do Trabalhador e Desigualdades em Saúde, \\ Universidade Estadual do Sudoeste da Bahia, Jequié, Bahia \\ *** Mestre em Saúde Coletiva, Professora Assistente, Pesquisadora do Núcleo de Estudo e Pesquisa em Saúde do \\ Trabalhador e Desigualdades em Saúde, Universidade Estadual do Sudoeste da Bahia, Jequié, Bahia \\ **** Mestre em Saúde Coletiva, Pesquisador do Núcleo de Estudo e Pesquisa em Saúde do Trabalhador e Desigualdades \\ em Saúde, Universidade Estadual do Sudoeste da Bahia, Jequié, Bahia \\ ***** Doutoranda em Medicina e Saúde Humana, Mestre em Saúde Coletiva, Professora Assistente, Coordenadora e \\ Pesquisadora do Núcleo de Estudo e Pesquisa em Saúde do Trabalhador e Desigualdades em Saúde, Universidade Estadual \\ do Sudoeste da Bahia, Jequié, Bahia
}

\section{Resumo}

Objetivo: Este estudo buscou descrever o perfil dos acidentes de trabalho segundo a causa e o desfecho na Região Sudoeste da Bahia. Metodologia: Trata-se de uma investigação com delineamento transversal acerca das Comunicações de Acidente de Trabalho preenchidas no Centro de Referência em Saúde do Trabalhador de Jequié, na região Sudoeste da Bahia, no período de Janeiro de 2006 a Dezembro de 2008. Resultados: Entre os 212 registros encontrados, $71,7 \%$ corresponderam a indivíduos do sexo masculino e $64,1 \%$ a faixa etária entre 21 a 40 anos. A natureza do acidente mais frequente foi o impacto por objeto $(24,8 \%)$ enquanto as fraturas $(22,4 \%)$ e contusões $(17,9 \%)$ predominaram entre as lesões. Conclusão: Apesar do expressivo número de acidentes registrados, as sub-notificações e os sub-registros persistem. No entanto, acredita-se que a implementação de ações de Vigilância a Saúde do Trabalhador possam minimizar esta lacuna, assim como, prevenir possíveis acidentes de trabalho. Palavras-chave: Acidentes de Trabalho; Notificação de Acidentes de Trabalho; Sistemas de Informação. 


\title{
PROFILE OF OCCUPATIONAL ACCIDENTS IN THE SOUTHWEST REGION OF BAHIA
}

\begin{abstract}
Objective: This study sought to describe the profile of workplace accidents by cause and outcome in southwest Bahia. Methodology: It is a cross-sectional investigation about Communications of Occupational Accident filled in the Reference Centre for Occupational Health in this region, in the period of January 2006 to December 2008. Results: Among the 212 records found, $71.7 \%$ corresponded to males and $64.1 \%$ aged between $21-40$ years. The nature of the most frequent accident was the impact by object (24.8\%) while the fractures $(22.4 \%)$ and contusions (17.9\%) predominated among the lesions. Conclusion: Despite the significant number of reported accidents, the sub-reports and sub-records remain. However, it is believed that the implementation of Health Workers Surveillance actions can minimize this gap, as well as prevent possible accidents.

Keywords: Occupational Accidents; Occupational Accident Communication; Health Information Systems; Health Workers Surveillance.
\end{abstract}

\section{INTRODUÇÃO}

Após a III Conferência Nacional de Saúde do Trabalhador, uma afirmativa ficou em evidência, "trabalhar sim, adoecer não!", esta declaração se deu por conta do crescimento acentuado das estatísticas relacionadas aos Acidentes de Trabalho (AT). O coeficiente médio de mortalidade, no período de 1999 a 2003, foi de 14,84 por 100.000 trabalhadores. A comparação desse coeficiente com o de outros países - Finlândia, 2,1 (2001); França, 4,4 (2000); Canadá 7,2 (2002); e Espanha, 8,3 (2003) - demonstra que o risco de morrer por acidente de trabalho, no Brasil, é cerca de duas a sete vezes maior. ${ }^{(1)}$

Segundo a Lei $n^{\circ} 8.213$, de 24 de Julho de 1991 (Lei da Previdência Social), em seu artigo 19, o acidente de trabalho é definido como "o que ocorre pelo exercício do trabalho a serviço da empresa [...] provocando lesão corporal ou perturbação funcional que cause morte ou a perda ou redução, permanente ou temporária, da capacidade para o trabalho". O artigo 22 da Lei citada, estabelece a notificação do acidente de trabalho obrigando o empregador, sob pena de multa, a comunicar à Previdência Social a ocorrência do acidente de trabalho através da emissão da Comunicação de Acidente de Trabalho (CAT). Esta mesma Lei afirma que "na falta de comunicação por parte da empresa, podem formalizá-la o próprio acidentado, seus dependentes, a entidade sindical competente, o médico que o assistiu ou qualquer autoridade pública". ${ }^{(2)}$

Segundo as estatísticas do Ministério da Previdência Social, durante o ano de 2007, foram registrados no Instituto Nacional de Seguridade Social (INSS) cerca de 653,1 mil acidentes do trabalho no Brasil. Em comparação com ano de 2006, o número de acidentes de trabalho aumentou 27,5\%. Estes achados não são exclusivos do Brasil, pois em todo o mundo os acidentes de trabalho são considerados um problema de saúde pública por serem potencialmente 
fatais, incapacitantes e acometerem, em especial, pessoas jovens e em idade produtiva, o que acarreta grandes consequências sociais e econômicas. ${ }^{(3)}$

Nesse contexto, os acidentes de trabalho geram influências econômicas e sociais, representando perdas que acometem trabalhadores, empregadores e o governo, já que os últimos são os responsáveis pela seguridade do trabalhador após o trauma. Os AT podem ser demasiadamente lesivos para o trabalhador quando se leva em consideração a causa e o desfecho do acidente, podendo ser fatais ou não. Contudo, os AT são passíveis de prevenção, sendo de grande importância conhecer as características destes.

A verdadeira situação dos acidentes de trabaIho no Brasil, não é totalmente conhecida, um dos motivos se dá provavelmente em relação às fontes de informação, pois os dados obtidos pela Previdência Social são os principais indicadores das estatísticas oficiais através da Comunicação de Acidente de Trabalho (CAT), que incluem apenas os trabalhadores com carteira de trabalho assinada, excluindo dessa forma os trabalhadores do setor informal.(4)

Neste contexto, a proposta deste estudo é descrever o perfil dos acidentes de trabalho segundo a causa e o desfecho, entre trabalhadores da região sudoeste da Bahia.

\section{MATERIAIS E MÉTODOS}

Trata-se de um estudo de corte transversal, cuja população foi composta pelos registros dos acidentes de trabalho ocorridos na região sudoeste da Bahia, Brasil, notificados no Centro de Referência em Saúde do Trabalhador (CEREST), da rede Sistema Único de Saúde (SUS) desta região, no período de Janeiro de 2006 a Dezembro de 2008.

A coleta de dados foi realizada por meio de um instrumento padronizado composto por três blocos de questões: a) Características sociodemográficas: sexo, idade, estado civil e área de residência; b) Características ocupacionais: ocupação, empresa, município da empresa, renda individual; c) Carac- terísticas do acidente: perfil do acidente, tipo, data, hora do acidente, município, local, natureza do acidente e/ou da doença, natureza da lesão, tipo de doença, parte do corpo atingida, emitente e tipo de CAT. O mesmo foi oriundo de dados contidos nas Comunicações de Acidentes de Trabalho arquivadas no CEREST-Jequié.

Foram estudadas as seguintes variáveis: a) causa: sexo, idade, estado civil, área de residência, ocupação, renda e natureza da doença; b) desfecho: tipo do acidente, perfil do acidente, mês do acidente, turno do acidente, ano do acidente, local do acidente, natureza do acidente, natureza da lesão, doença e parte do corpo atingida. Estas variáveis foram selecionadas a partir dos campos da CAT referentes aos potenciais fatores causais e o desfecho dos acidentes de trabalho, portanto, não houve inferência causal.

O instrumento foi utilizado para a transferência dos dados existentes na CAT, que após coletados foram transferidos para o software Excel $2007^{\circledR}$ e o programa Epilnfo Versão $6.044^{\circledR}$, para reconhecer os arquivos gerados, posteriormente analisados. Os dados foram analisados através da estatística descritiva, onde foram estimadas frequências relativas e absolutas.

O estudo é um recorte do Projeto-mãe intitulado "Perfil dos acidentes de trabalho na região sudoeste da Bahia, Brasil". Foi apreciado e aprovado pelo Comitê de Ética em Pesquisa da Universidade Estadual do Sudoeste da Bahia (CEP-UESB), protocolo $n^{\circ}$ 112-2008, de acordo com as normas da Resolução 196/96, do Conselho Nacional de Saúde, que regula Pesquisa em Seres Humanos.

\section{RESULTADOS}

Foram analisadas 212 CAT referentes ao período de janeiro/2006 a dezembro/2008, que se encontravam no CEREST-Jequié. Observou-se predominância do sexo masculino ( $71,7 \%)$; sendo que a faixa etária mais expressiva foi entre 21-40 anos $(64,1 \%)$. O estado civil que concentrou o maior número de acidentes foi o de casados $(52,4 \%)$, 
seguida de solteiros $(42,0 \%)$. Com relação à renda mensal, 56,6\% recebiam entre $R \$ 301,00$ e $R \$$ 500,00 . Observou-se que $87,7 \%$ das vítimas residiam na cidade de Jequié e a maioria não era aposentada (99,5\%). Em relação à área de residência, evidenciou-se que há maior concentração de acidentes de trabalho entre moradores da zona urbana (90,6\%) (Tabela 1).

Tabela 1- Caracterização da população segundo variáveis sociodemográficas. Jequié, Bahia, 2009. (N=212)

\begin{tabular}{lcc}
\hline VARIÁVEIS & $\mathrm{N}$ & $(\%)$ \\
\hline Sexo & 152 & 71,7 \\
Masculino & 60 & 28,3 \\
Feminino & & \\
Idade & 4 & 1,9 \\
$<20$ anos & 137 & 64,6 \\
$21-40$ anos & 70 & 33,0 \\
$41-60$ anos & 1 & 0,5 \\
$>61$ anos & & \\
Estado Civil & 111 & 52,4 \\
Casado & 89 & 42,0 \\
Solteiro & 2 & 0,9 \\
Viúvo & 10 & 4,7 \\
Não informado & & \\
Renda Mensal & 5 & 2,4 \\
10O-3OO & 120 & 56,6 \\
3Ol-5OO & 18 & 8,5 \\
5Ol-7OO & 7 & 3,3 \\
7Ol-9OO & 25 & 11,8 \\
$>$ 9OO & 37 & 17,5 \\
Não informado & & \\
Área de residência & 192 & 90,6 \\
Urbana & 20 & 9,4 \\
Rural & & \\
\hline
\end{tabular}

De acordo com os achados, dentre as ocupações que foram mais acometidas pelos AT, destaca-se trabalhadores da confecção de calçados e auxilia- res diversos $(14,2 \%)$, os trabalhadores da construção civil $(12,7 \%)$ e os trabalhadores rurais $(8,0 \%)$ (Tabela 2).

Tabela 2 - Caracterização da população segundo variável ocupacional. Jequié, Bahia, 2009. (N=212)

\begin{tabular}{lcc}
\hline VARIÁVEIS & $\mathbf{N}$ & (\%) \\
\hline Trabalhador rural & 17 & 8,0 \\
Trabalhador pólo calçadista & 30 & 14,2 \\
Auxiliares & 30 & 14,2 \\
Motorista & 12 & 5,7 \\
Operador & 13 & 6,1 \\
Trabalhador da construção civil & 27 & 12,7 \\
Vendedor & 13 & 6,1 \\
Outros & 70 & 33,0 \\
\hline
\end{tabular}


Quanto ao tipo de acidente, os considerados típicos foram mais frequentes ( $47,6 \%)$, seguido dos acidentes de trajeto $(28,3 \%)$. As doenças ocupacionais corresponderam à $24,1 \%$ dos registros. Quanto ao perfil, a maioria do desfecho foi não-fatal (99,5\%). No que se refere ao período de ocorrência, $33,4 \%$ aconteceram no último quadrimestre anual, sendo que o ano de 2008 foi o que apresentou o maior número de Comunicações $(39,6 \%)$, seguido do ano de 2007 (31,1\%). Dentre os acidentes de trabalho, 41,5\% ocorreram no turno da manhã. As partes do corpo que predominaram nos acometimentos foram os membros superiores $(49,1 \%)$ seguido dos membros inferiores $(20,8 \%)$ (Tabela 3 ).

Tabela 3 - Caracterização da população segundo variáveis perfil do acidente. Jequié, Bahia, 2009. (N=212)

\begin{tabular}{|c|c|c|}
\hline VARIÁVEIS & $\mathrm{N}$ & $(\%)$ \\
\hline \multicolumn{3}{|l|}{ Tipo de acidente } \\
\hline Típico & 101 & 47,6 \\
\hline Trajeto & 60 & 28,3 \\
\hline Doença ocupacional & 51 & 24,1 \\
\hline \multicolumn{3}{|l|}{ Perfil do acidente } \\
\hline Fatal & 1 & 0,5 \\
\hline Não fatal & 211 & 99,5 \\
\hline \multicolumn{3}{|l|}{ Mês do acidente } \\
\hline Janeiro-Abril & 51 & 24,0 \\
\hline Maio-Agosto & 67 & 31,7 \\
\hline Setembro a dezembro & 71 & 33,4 \\
\hline Não informado & 23 & 10,8 \\
\hline \multicolumn{3}{|l|}{ Ano do acidente } \\
\hline 2006 & 46 & 21,7 \\
\hline 2007 & 66 & 31,1 \\
\hline 2008 & 84 & 39,6 \\
\hline Outros & 13 & 6,2 \\
\hline Não informado & 3 & 1,4 \\
\hline \multicolumn{3}{|l|}{ Turno do acidente } \\
\hline Manhã & 88 & 41,5 \\
\hline Tarde & 53 & 25,0 \\
\hline Noite & 8 & 3,8 \\
\hline Madrugada & 11 & 5,2 \\
\hline Não informado & 52 & 24,5 \\
\hline \multicolumn{3}{|l|}{ Parte do corpo } \\
\hline MMSS & 104 & 49,1 \\
\hline MMII & 44 & 20,8 \\
\hline Coluna vertebral & 20 & 9,4 \\
\hline Cabeça & 4 & 1,9 \\
\hline Múltiplas & 23 & 10,8 \\
\hline Outras & 17 & 8,0 \\
\hline
\end{tabular}


Em todos os acidentes típicos e de trajeto (161), o impacto por objeto $(24,8 \%)$ foi a natureza do acidente mais frequente, seguido por quedas e acidentes automobilísticos, ambos com $17,4 \%$. Quanto à natureza da lesão, predominaram as fraturas $(22,4 \%)$ e as contusões $(17,9 \%)$. No total de acidentes enquadrados como doença ocupacional a atividade manual repetitiva foi a natureza da doença mais observada $(66,0 \%)$. A LER/DORT foi a doença mais frequente $(51,8 \%)$, seguida da hérnia discal $(12,5 \%)$ (Tabela 4). Em se tratando do local do acidente de trajeto, $73,3 \%$ aconteceram em ruas e vias públicas. No que se refere aos tipos de CAT, 70,3\% eram iniciais e $78,3 \%$ foram emitidas pelo empregador.

Tabela 4 - Caracterização da população segundo variáveis natureza do acidente e da lesão. Jequié, Bahia, 2009. $(\mathrm{N}=212)$

\begin{tabular}{|c|c|c|}
\hline VARIÁVEIS & $N$ & (\%) \\
\hline \multicolumn{3}{|l|}{ Natureza do acidente } \\
\hline Atropelamento & 10 & 4,7 \\
\hline Carregamento de peso & 1 & 0,5 \\
\hline Impacto por objeto & 40 & 18,9 \\
\hline Queda & 28 & 13,2 \\
\hline Queda de altura & 19 & 9,0 \\
\hline Acidente automobilístico & 28 & 13,2 \\
\hline Outros & 32 & 15,1 \\
\hline Não informado & 54 & 25,5 \\
\hline \multicolumn{3}{|l|}{ Natureza da lesão } \\
\hline Contusão & 28 & 13,2 \\
\hline Corte & 14 & 6,6 \\
\hline Decepação & 7 & 3,3 \\
\hline Entorse & 5 & 2,4 \\
\hline Fratura & 35 & 16,5 \\
\hline Queimadura & 9 & 4,2 \\
\hline Inalação de substâncias & 11 & 5,2 \\
\hline Múltiplas & 24 & 11,3 \\
\hline Outras & 23 & 10,8 \\
\hline Não informado & 56 & 26,4 \\
\hline \multicolumn{3}{|l|}{ Natureza da doença } \\
\hline Atividade manual repetitiva & 37 & 17,5 \\
\hline Manutenção da posição sentada & 8 & 3,8 \\
\hline Exposição a agentes químicos & 1 & 0,5 \\
\hline Inalação de substâncias & 2 & 0,9 \\
\hline Outros & 3 & 1,4 \\
\hline Não informado & 161 & 75,9 \\
\hline \multicolumn{3}{|l|}{ Doença } \\
\hline Hérnia de disco & 7 & 3,3 \\
\hline LER/DORT & 29 & 13,7 \\
\hline Dermatose & 1 & 0,5 \\
\hline Asma ocupacional & 2 & 0,9 \\
\hline Outras & 10 & 4,7 \\
\hline Não informado & 163 & 76,9 \\
\hline
\end{tabular}




\section{DISCUSSÃO}

Os AT acometeram principalmente trabalhadores do sexo masculino, jovens, residentes em zona urbana, trabalhadores do pólo calçadista e auxiliares, vítimas de acidentes típicos e desfechos não fatais, acometendo principalmente membros superiores.

Estudos corroboram com esse achado, demonstrando a caracterização da população atingida que acompanha o perfil observado em outras regiões do Brasil: homens jovens em idade produtiva. ${ }^{(5,6)} \mathrm{Em}$ bora seja evidente esta caracterização, um estudo acerca da incidência anual de acidentes de trabalho não fatais, afirma que mesmo havendo uma incidência maior entre os homens $(6,05 \%)$ do que entre as mulheres $(5,53 \%)$, tal diferença não foi estatisticamente significante. ${ }^{(4)}$

O predomínio de indivíduos casados acometidos pelos AT também foi um achado de outro estudo, $(63,16 \%)$. Acredita-se que este número elevado deve-se à função de sustento da família, assim como, a contribuição das mulheres casadas na composição da renda familiar. ${ }^{(4,7)} \mathrm{Em}$ um estudo para associar as características individuais e sociodemográficas dos acidentes em Pelotas-RS, observou-se também um maior numero de acidentes em trabaIhadores casados. ${ }^{\left({ }^{8}\right)}$

Uma proporção elevada de indivíduos apresentou renda mensal individual entre $\mathrm{R} \$ 301,00$ e $\mathrm{R} \$$ 500,00 . Estes valores se enquadram nas alterações salariais feitas nos últimos anos, equivalendo a cerca de "1 a 2 salários"; confirmando achados de outro estudo, o qual demonstra que os acidentes foram frequentes entre as três primeiras classes de renda, isto é, entre "o,o e 2,o salários mínimos".(9) Tal achado corrobora também com um estudo em que a renda familiar foi inferior a dois salários mínimos mensais para $16 \%$ dos acidentados; e nestes casos, o número de acidentes em trabalhadores de baixa renda foi três vezes maior que os com renda superior ou igual a seis salários mínimos. ${ }^{(8)}$ Este número está diretamente associado à inserção dos trabalhadores em setores/ramos de atividade com menor remuneração, no qual exige-se menor nível de escolaridade, e apresentam piores condições de trabalho.

Dentre as ocupações mais acometidas, as que apresentaram maior frequência de acidentes foram: os trabalhadores do pólo calçadista, auxiliares diversos, trabalhadores da construção civil seguido dos trabalhadores rurais. Este achado condiz com o estudo sobre o perfil epidemiológico dos acidentes de trabalho no Vale dos Sinos, a estreita ligação e a grande importância dos setores coureiro-calçadista e têxtil quanto à morbidade ocupacional na região, principalmente em relação aos trabalhadores do setor de produção. As repercussões para os trabalhadores deste setor se dão principalmente devido à realização de atividades repetitivas por longos períodos, ocasionando muitas vezes incapacidades fisicas evoluídas para doenças ocupacionais. Ainda neste estudo identificou-se que $90,2 \%$ dos acidentes de trabalho ocorreram na zona urbana e $1,1 \%$ na zona rural; (5) pois a maioria das funções que acarretam mais riscos, como é o caso do setor de construção civil e eletrificação, apresentam uma abordagem mais urbana.

Evidências afirmam que o ramo de atividade econômica de maior risco de acidentes fatais foi à construção civil em Campinas. ${ }^{(10)}$ Ramo, cujos trabalhadors têm menor nível de escolaridade, e maior vulnerabilidade. Este setor absorve maior contingente de mão de obra e participam de várias etapas no processo produtivo, ao tornar mais expostos aos riscos, Assim como, muitos trabalhadores nem sempre estão orientados sobre o uso de EPIs, ampliando o risco e a gravidade do acidente.

Quanto ao período de ocorrência dos acidentes observa-se que o ano de 2008 apresentou um maior número de casos, dado este que pode ser justificado pelas ações realizadas pelo CEREST no referido ano de incentivo local para a notificação dos acidentes. Portanto, os achados observados não necessariamente significam o aumento no número dos acidentes, mas pode estar revelando uma característica comum em outros estudos que é o sub-registro de acidentes..$^{(7,9-11)}$ 
Em relação ao período de ocorrência verifica-se um expressivo número de acidentes aconteceram no turno da manhã, condizendo com achados de outro estudo, onde observou-se que $49,1 \%$ dos acidentes ocorreram nesse período. ${ }^{(12)}$ Encontrou-se maior proporção de acidentes de trabalho ocorridos durante o período da manhã $(44,99 \%) ;{ }^{(13)}$ pois o período da manhã geralmente apresenta maior sobrecarga de trabalho em diversos setores ocupacionais, ocasionando desta forma uma frequência tão alta de acidentes.

Dentre as partes do corpo atingidas pelo acidente, os membros superiores foram os que apresentaram uma frequência mais acentuada assim como observado em um estudo retrospectivo, onde os membros superiores foi a parte do corpo mais acometida em $61,14 \%$ dos acidentes. ${ }^{(13)}$ Identificou-se achado parecido em um estudo mediante análise de prontuários hospitalares $(30,7 \%) .{ }^{(14)}$ Os membros superiores são as partes do corpo mais diretamente envolvidas com determinados tipos de trabalho e, consequentemente, mais expostas ao acidente; em ramos específicos, como o da construção civil e o setor calçadista em que a atividade com membros superiores é comum. ${ }^{(15)}$

A natureza do acidente mais registrada foi o impacto por objeto seguido de acidentes automobilísticos; já a natureza da lesão mais acentuada foram as fraturas. ${ }^{(15)}$ Tais osachados são confirmados também, em um estudo retrospectivo sobre acidentes automobilísticos e a natureza da lesão. ${ }^{(16)}$ Levando-se em consideração que impactos diretos, ou indiretos de alta energia e velocidade, como no caso de veículos motores, a existência de fraturas é um achado comum, explicando dessa forma a presença tão significativa deste dado; deve ser lembrado que em setores específicos o não uso de equipamentos de proteção individual podem potencializar ainda mais a gravidade do acidente. ${ }^{(3,17,18)}$

Em relação aos dados de doenças ocupacionais, a natureza da doença mais frequente foi a atividade manual repetitiva. A doença predominante foi a LER/DORT, este dado está intimamente ligado com o exposto anteriormente segundo este mesmo es- tudo. Considerando as doenças ligadas ao apareIho locomotor como a terceira mais frequente, após Hipertensão Arterial e Transtornos Mentais. ${ }^{(19,20)}$ A atividade manual repetitiva e as LER/DORT foram identificadas principalmente nos tipos de acidente enquadrados como doença ocupacional, principalmente, os acometimentos de trabalhadores do ramo calçadista, submetidos a cargas de trabalho muitas vezes sem descanso e a manutenção de posturas inadequadas para o trabalho, apresentando frequências elevadas.

\section{CONSIDERAÇÕES FINAIS}

Os dados atuais sobre os acidentes na Região Sudoeste da Bahia demonstram de forma discreta a real situação dos trabalhadores, porque ainda não existe uma notificação adequada, problema presente em todo o país, a subnotificação e dificuldade de integração dos bancos de dados oficiais. ${ }^{(7,21,22,23)}$

Apesar do expressivo número de acidentes registrados, as sub-notificações e os sub-registros persistem. No entanto, acredita-se que a implementação de ações de Vigilância à Saúde do Trabalhador possam minimizar esta lacuna, assim como, prevenir possíveis acidentes de trabalho.

Agradecimentos: Os autores agradecem o apoio dos técnicos do Centro de Referência Regional em Saúde do Trabalhador-CEREST, do município de Jequié, Bahia.

\section{REFERÊNCIAS}

1. Brasil. Ministério da Saúde. Conferência Nacional de Saúde do Trabalhador - Tema: "Trabalhar Sim, Adoecer Não". Brasília; 2008 [acesso 17 out. 2008]. Disponível em: http://bvsms.saude.gov.br/bvs/trabalhador/ conferencias.html

2. Brasil. Lei no 8.213 , de 24 de julho de 1991. Dispõe sobre os planos de benefícios da previdência social e dá outras providências. Diário Oficial [da] República Federativa do Brasil, Brasília, DF, 14 ago. 1998. In: SISLEX: 
Sistema de Legislação, Jurisprudência e Pareceres da Previdência e Assistência Social. [S.I.]: DATAPREV, 1999 [acesso 25 set. 2008]. Disponível em: http://wwwO1O.dataprev.gov.br/ sislex/

3. Lucca SR, Mendes R. Epidemiologia dos acidentes do trabalho fatais em área metropolitana da região sudeste do Brasil, 19791989. Rev. saúde pública; 1993; 27(3):168-176.

4. Santana V, Maia AP, Carvalho C, Luz G. Acidentes de trabalho não fatais: diferenças de gênero e tipo de contrato de trabalho. Cad. saúde pública. 2003; 19(2):481-493.

5. Hennington EA, Monteiro MO. Perfil epidemiológico dos acidentes de trabalho no Vale dos Sinos e o sistema de vigilância em saúde do trabalhador. Hist. ciênc. saúdeManguinhos. 2006;13(4):865-876.

6. Conceição PSA, Nascimento IBO, Oliveira PS, Cerqueira MRM. Acidentes de trabalho atendidos em serviço de emergência. Cad. saúde pública. 2003;19(1):111-117.

7. Binder MCP, Cordeiro R. Sub-registro de acidentes do trabalho em localidade do Estado de São Paulo, 1997. Rev. saúde pública. 2003;37(4):409-416.

8. Lima RC, Victora CG, Dall'Agnol M, Facchini LA, Fassa A. Associação entre as características individuais e sócio-econômicas e os acidentes do trabalho em Pelotas, Rio Grande do Sul, Brasil. Cad. saúde pública. 1999; 15(3): 569-580.

9. Barata RCB, Ribeiro MCSA, Moraes JC. Acidentes de trabalho referidos por trabalhadores moradores em área urbana no interior do Estado de São Paulo em 1994. Inf. epidemiol. SUS. 2000;9(3):199-210.

1O. Santana VS, Nobre L, Waldvogel B. Acidentes de trabalho no Brasil entre 1994 e 2004: uma revisão. Ciênc. saúde coletiva. 2005;10(4):84155.

11. Santana VS, Araújo-Filho JB, Silva M, Oliveira PRA, Branco AB, Nobre LCC. Mortalidade, anos potenciais de vida perdidos e incidência de acidentes de trabalho na Bahia, Brasil. Cad. saúde pública. 2007;23(11):2643-2652.

12. Souza M, Vianna LAC. Incidência de acidentes de trabalho relacionada com a não utilização das precauções universais. Rev. bras. enferm. 1993;46:234-44.

13. Miranzi SSC, Gaspar AACS, Iwamoto HH, Miranzi MASM, Dziabas DC. Acidentes de trabalho entre os trabalhadores de uma universidade pública. Rev. bras. saúde ocup. 2008;33 (118):40-47.

14. Silveira CA, Robazzi MLCC; Walter EV, Marziale MHP. Acidentes de trabalho na construção civil identificados através de prontuários hospitalares. REM: Rev. Esc. Minas. 2005;58(1):39-44.

15. Ferreira RR, Mendes R. Alguns aspectos epidemiológicos dos acidentes de trabalho fatais ocorridos em Campinas, SP (Brasil), 19721978. Rev. saúde pública. 1981;15(3):251-262.

16. Sallum AMC, Poizumi MS. Natureza e gravidade das lesões em vítimas de acidente de trânsito de veiculo a motor. Rev. Esc. Enferm. USP. 1999;33(2)157-64.

17. Conceição PSA, Nascimento IBO, Oliveira PS,Cerqueira MRM. Acidentes de trabalho atendidos em serviço de emergência. Cad. saúde pública. 2003; 19(1)111-117.

18. Machado JMH, Gomez CM. Acidentes de trabalho: uma expressão da violência social. Cad. saúde pública.1994;10(1):74-87.

19. Mendes RO. Impacto dos efeitos da ocupação sobre a saúde de trabalhadores: I. Morbidade. Rev. saúde pública. 1988; 22(4): 311-326.

2O. Filho VW. Perfil epidemiológico dos trabalhadores. Rev. bras. med. trab. 2004; 2:103-117.

21. Teixeira MLP, Freitas RMV. Acidentes do trabalho rural no interior paulista. São Paulo perspect. 2003;17(2):81-90.

22. Correa PRL, Assunção AAA. Subnotificação de mortes por acidentes de trabalho: estudo de três bancos de dados. Epidemiol. Serv. Saúde. 2003;4(12):203-212.

23. Brasil. Ministério da Previdência Social. Anuário Estatístico da Previdência Social 2007: Seção IV - Acidentes do Trabalho. Brasília; 2007 [acesso 30 out. 2008]. Disponível em: http:// www.previdencia.gov.br/aeps2007/16_01_03_01. asp 